\title{
EphA3 contributes to tumor growth and angiogenesis in human gastric cancer cells
}

\author{
XIAO-YE LV $^{1,2^{*}}$, JIAN WANG ${ }^{1 *}$, FANG HUANG ${ }^{1}$, PENG WANG $^{1}$, JIAN-GUANG ZHOU $^{1}$, \\ $\mathrm{BO} \mathrm{WEI}^{2}$ and SHAN-HU LI ${ }^{1}$ \\ ${ }^{1}$ Department of Cell Engineering, Beijing Institute of Biotechnology, Beijing 100850; \\ ${ }^{2}$ Department of General Surgery, The General Hospital of People's Liberation Army, Beijing 100853, P.R. China
}

Received December 7, 2017; Accepted July 13, 2018

DOI: $10.3892 / o r .2018 .6586$

\begin{abstract}
Eph receptor tyrosine kinases and their ephrin ligands, mediate an important cell communication system both in normal and oncogenic development, and play central roles in a series of processes including angiogenesis, stem cell maintenance and cancer metastasis. Eph receptor A3 (EphA3), commonly overexpressed in a broad range of cancers, including gastric cancer (GC), is related to tumor progression. Our previous study revealed that EphA3 may play important roles in tumorigenesis and angiogenesis in GC. However, its exact role and the mechanisms underlying its function in $\mathrm{GC}$ remain unclear. In the present study, lentivirus-mediated RNA interference was employed to knock down the expression of EphA3 in GC HGC-27 cells. Functional analyses indicated that depletion of EphA3 expression inhibited the cell growth and tumorigenicity of HGC-27 cells in vitro and in vivo. Furthermore, knockdown of the expression of EphA3 in HGC-27 cells inhibited tube formation and migration of HUVEC endothelial cells. Tumor angiogenesis in vivo was also inhibited upon EphA3 knockdown in HGC-27 cells, with reduced microvessel density (MVD) in xenograft models. We further revealed that EphA3 depletion inhibited tumor angiogenesis and migration through the signal transducer and activator of transcription 3/vascular endothelial growth factor (STAT3/VEGF) signaling pathway. These results indicated
\end{abstract}

Correspondence to: Dr Shan-Hu Li, Department of Cell Engineering, Beijing Institute of Biotechnology, 27 Taiping Road, Beijing 100850, P.R. China

E-mail: lishanhu6@163.com

Dr Bo Wei, Department of General Surgery, The General Hospital of People's Liberation Army, 28 Fuxing Road, Beijing 100853, P.R. China

E-mail: weibo@vip.163.com

${ }^{*}$ Contributed equally

Key words: EphA3, gastric cancer, HGC-27, angiogenesis, vascular endothelial growth factor that EphA3 may be an effective prognostic indicator and a potential target for GC therapy.

\section{Introduction}

Gastric cancer (GC) remains one of the most common cancers and the third leading cause of tumor-related deaths (1). Seventy percent of the newly detected cases and deaths from GC were in developing countries, of which $42 \%$ occurred in China (2). Despite advances in the treatment of GC, the prognosis of GC remains poor. Like all other cancers, the development of GC is a multistep process involving numerous genetic and epigenetic alterations of growth factors and receptors, angiogenic factors, cell cycle regulators and DNA mismatch repair genes (3). These multiple factors on genetic and epigenetic alterations are likely to be the target in GC cancer prevention and treatment. Therefore, identifying the molecular mechanisms underlying the development of GC can benefit diagnosis and treatment of this disease.

Eph receptors are the largest known subfamily of receptor tyrosine kinases that are activated in response to binding with Eph receptor-interacting proteins (ephrins). Eph receptors have been divided into two groups based on their sequence homology and their affinities for binding ephrin-A and ephrin-B ligands: EphA and EphB receptors. In the human genome, there are nine EphAs and five EphBs that generally bind preferentially to five ephrin-A and three ephrin-B ligands, respectively $(4,5)$. The Eph receptor and their ligands are frequently overexpressed in a variety of cancers and affect tumor growth, angiogenesis and metastasis (6). However, Eph signaling activities in cancer are complex and interesting in their paradoxical effects.

EphA3, an EphA receptor subfamily member, has been found to be aberrantly expressed in a variety of human cancers including malignant melanoma, glioblastoma and mutated in lung and breast cancer $(7,8)$. Increased expression of EphA3 can promote tumor cellular proliferation, angiogenesis, invasion and is regarded as a promising target in cancer therapy (9). Soluble EphA3-Fc receptors and the anti-EphA3 antibody can inhibit tumor angiogenesis and progression $(10,11)$. However, little is known about the function of EphA3 in GC. Our previous study reported that the upregulated expression of EphA3 in GC was correlated with tumor size, distant metastasis, pTNM 
stage and poor prognosis. We also observed that the expression of EphA3 was significantly and positively associated with VEGF and microvessel density (MVD) expression (12). In the present study, we extended our previous study and knocked down EphA3 in HGC-27 cells to investigate the direct role of EphA3 in GC cell growth and angiogenesis.

\section{Materials and methods}

Cell lines and culture conditions. Human GC cell line HGC-27 and human umbilical vein endothelial cells (HUVECs) were purchased from the Cell Bank of Shanghai Institute of Biochemistry and Cell Biology, Chinese Academy of Sciences (Shanghai, China). Human GC cell lines (AGS, SGC-7901 and MGC-803) and a human gastric epithelial cell line (GES-1) were purchased from the American Tissue Culture Collection (ATCC; Manassas, VA, USA). GC cells or HUVECs were grown in RPMI-1640 medium or Dulbecco's Modified Eagle's medium (DMEM; Sigma-Aldrich; Merck KGaA, Darmstadt, Germany) supplemented with $10 \%$ fetal bovine serum (FBS; HyClone Laboratories; GE Healthcare Life sciences, Chicago, IL, USA). All the media were supplemented with $100 \mathrm{U} / \mathrm{ml}$ penicillin and $100 \mu \mathrm{g} / \mathrm{ml}$ streptomycin (Invitrogen; Thermo Fisher Scientific, Inc., Waltham, MA, USA) and maintained in a $5 \% \mathrm{CO}_{2}$ incubator at $37^{\circ} \mathrm{C}$.

Reagents. Antibodies against STAT3 (1:1,000; cat. no. 9139), p-STAT3(Tyr705) (1:1,000; cat. no. 9145), AKT (1:1,000; cat. no. 4691) and p-AKT473 (1:1,000; cat. no. 4060; ) were purchased from Cell Signaling Technology (Danvers, MA, USA). Antibodies against EphA3 (1:200; cat. no. sc-919), CD31 (1:100; cat. no. sc-1506) and $\gamma$-tubulin (1:500; cat. no. sc-7396;) were obtained from Santa Cruz Biotechnology (Santa Cruz, CA, USA). VEGF neutralizing antibody (V4758; $2 \mu \mathrm{g} / \mathrm{ml}$ ) was purchased from Sigma-Aldrich; Merck KGaA. Growth factor-reduced Matrigel was obtained from BD Biosciences (Bedford, MA, USA). Medium 200PRF and low-serum growth supplements (LSGS) were obtained from Invitrogen; Thermo Fisher Scientific, Inc. A VEGF ELISA Development kit was purchased from PeproTech, Inc. (Rocky Hill, NJ, USA).

Lentiviral vector production and cell transduction. Stable knockdown of EphA3 in HGC-27 cells was obtained using lentivirus. In brief, pLKO.1-EphA3-shRNA or scrambled plasmids were co-transfected with psPAX2 and pMD2.G plasmids into $293 \mathrm{~T}$ cells at $70 \%$ confluency. Following $48 \mathrm{~h}$, viral supernatants were collected and filtrated. Subconfluent HGC-27 cells were transduced with lentivirus in the presence of $8 \mathrm{mg} / \mathrm{ml}$ Polybrene (Sigma-Aldrich; Merck KGaA). Between 16 to $24 \mathrm{~h}$ post-transduction, the cells were replaced with fresh medium. Following $48 \mathrm{~h}$, transduced cells were selected with puromycin at $2 \mu \mathrm{g} / \mathrm{ml}$ for 3 days.

Preparation of tumor cell-conditioned medium (CM). To prepare CM, target cells shNC-HGC-27 or shEphA3-HGC-27 were seeded and grew to $30-40 \%$ confluency. Growth medium was replaced with serum-free RPMI-1640 medium for $24 \mathrm{~h}$. Subsequenlty, CM was harvested when cells reached $60-80 \%$ confluency. $\mathrm{CM}$ was aliquoted and stored at $-80^{\circ} \mathrm{C}$ since it was not immediately used. In addition, shNC-HGC-27 or
shEphA3-HGC-27 cells were pretreated with DMSO or WP1066 $(5 \mu \mathrm{M})$ in serum-free culture medium for $24 \mathrm{~h}$. The supernatants were collected as CM.

Western blot analysis. Cells were rinsed with phosphate-buffered saline (PBS) and lysed in ice-cold RIPA buffer [40 mM HEPES (pH 7.4), 1\% Triton X-100, 0.1\% SDS, $100 \mathrm{mM} \mathrm{NaCl}, 5 \mathrm{mM}$ EDTA, $0.5 \%$ sodium deoxycholate and $1 \mathrm{mM}$ sodium orthovanadate] with protease and phosphatase inhibitors. The BCA Protein Assay kit (Thermo Fisher Scientific, Inc.) was used to quantitate the lysates. The same amount of protein was separated by $8-10 \%$ SDS-PAGE gel and then transferred onto a PVDF membrane (Millipore, Bedford, MA, USA). Following blocking with 5\% non-fat dry milk in Tris-buffered saline containing $0.1 \%$ Tween-20 (TBST), the membrane was first incubated overnight at $4{ }^{\circ} \mathrm{C}$ with anti-STAT3 antibody, anti-p-STAT3 (Tyr705) antibody, anti-AKT antibody, anti-p-AKT473 and anti-EphA3 antibody and the corresponding signals were detected with an enhanced chemiluminescence kit (Thermo Fisher Scientific, Inc., Waltham, MA, USA) following incubation with the appropriate HRP-conjugated secondary antibodies (1:5,000; cat. nos. ZB-2301 and ZB2305; Zhongshan Golden Bridge Biotechnology Co., Ltd., Beijing, China). Quantification of band densities on western blot films was performed using ImageJ 1.44 software (National Institutes of Health, Bethesda, MD, USA).

Cell proliferation assay. Cell proliferation was assessed using Cell Counting Kit-8 (CCK-8; Dojindo Molecular Laboratories, Kumamoto, Japan) according to the manufacturer's instructions. In brief, $\sim 2 \times 10^{3}$ shNC-HGC-27 and shEphA3-HGC-27 cells were seeded into 96-well plates and incubated for the indicated time periods. Subsequently, $10 \mu \mathrm{l}$ of CCK- 8 solution was added to each well and incubated for $1 \mathrm{~h}$. The absorbance was finally determined at a wavelength of $450 \mathrm{~nm}$ with a microplate reader (Bio-Rad Laboratories Inc., Hercules, CA, USA). Each measurement was performed in triplicate and the experiments were repeated 3 times.

Colony formation assays. Cells were seeded into 6-well plates at a density of 1,000, 2,000 and 3,000 cells/well. Following culture for 14 days in complete growth media, the cells were washed with PBS and stained with crystal violet. Colonies containing 50 or more cells were counted under a light microscope at x40 magnification. Each measurement was performed at least three times.

ELISA assay. Secreted VEGF in CM of shNC-HGC-27 and shEphA3-HGC-27 was determined using a commercially available human VEGF-A ELISA Development kit (PeproTech) according to the manufacturer's instructions. The data were adjusted by the amount of total protein within the cells.

In vitro tube formation assay. A 96-well plate was coated with $50 \mu \mathrm{l}$ growth factor-reduced Matrigel and maintained at $37^{\circ} \mathrm{C}$ for $2 \mathrm{~h}$. Then, growth factor-deprived HUVECs suspended in $100 \mu 1$ of $\mathrm{CM}$ were plated at $1 \times 10^{4}$ cells/well and incubated at $37^{\circ} \mathrm{C}$ and $5 \% \mathrm{CO}_{2}$ for 4 to $6 \mathrm{~h}$ to allow the formation of a tubular structure. Cells were imaged and the tube lengths 
were counted using the Scion Image Beta 4.02 software (Scion Corp., Fredrick, MD, USA).

In vitro migration assay. A cell migration assay was performed using 24-well Boyden chambers with $8.0-\mu \mathrm{m}$ pore sizes (Corning, Inc., Corning, NY, USA). HUVECs were plated at $5 \times 10^{4}$ cells/well in the upper chamber of serum-free medium. The lower chamber was filled with $500 \mu \mathrm{l} \mathrm{CM}$ from shNC-HGC-27 or shEphA3-HGC-27 cells. The plates were incubated for 4 to $6 \mathrm{~h}$ at $37^{\circ} \mathrm{C}$ in $5 \% \mathrm{CO}_{2}$ and non-migrated cells were wiped away with cotton swabs. The cells that migrated to the lower side of the filter were fixed in $4 \%$ formaldehyde solution for $15 \mathrm{~min}$ and stained with $0.05 \%$ crystal violet in PBS for $15 \mathrm{~min}$. Migrated cells were counted using 10 random fields with an inverted phase contrast microscope. Filters were counted in triplicate per experiment. For VEGF inhibition assay, HUVEC migration ability was determined as aforementioned after adding neutralizing antibody $(0.2 \mu \mathrm{g} / \mathrm{ml})$ to VEGF-A into CM.

Xenograft models and immunohistochemistry. Five-week-old female BALB/c nude mice were purchased from Beijing Vital River Laboratory Animal Technology Co., Ltd. (Beijing, China). Three mice were housed per cage under controlled temperature and humidity $\left(23 \pm 2^{\circ} \mathrm{C}, 50 \pm 10 \%\right)$, on a 12 -h light/dark cycle. Mice had access to tap water and food $a d$ libitum. All animal studies conformed to the relevant regulatory standards and were approved by the Institutional Animal Care Committee of Beijing Institute of Biotechnology. Approximately $5 \times 10^{6}$ shNC-HGC-27 or shEphA3-HGC-27 cells were suspended in $200 \mu 1$ of serum-free RPMI-1640 medium and implanted subcutaneously into the right flank of 5-week-old female BALB/c nude mice $(n=3)$ separately. Tumor volumes were determined using digital calipers every 3 days and calculated as follows: length $\mathrm{x}$ width $\mathrm{x}$ height x 0.5236 . Mice were monitored for 3 weeks, when the experiment was terminated. Further termination points of the experiment were when the maximum tumor volume was $\sim 2 \mathrm{~cm}^{3}$ or when the mice showed any signs of distress (e.g., breathing disorders, weight loss, or immobility). At the end of the experiment the mice were anesthetized with $1.5 \%$ isofluorane-air mixture and sacrificed by cervical dislocation. Tumor xenografts were removed and prepared for immunohistochemistry. Tumor sections from shNC and shEphA3-HGC-27 mice were processed for immunohistochemical analysis of CD31 to visualize MVD. A portion of the tumor tissue was fixed in 10\% neutral-buffered formalin, dehydrated, embedded in paraffin and sectioned at 4- to 5- $\mu \mathrm{m}$ thickness. The sections were deparaffinized, treated with $3 \%$ $\mathrm{H}_{2} \mathrm{O}_{2}$ for $15 \mathrm{~min}$, and then were microwaved in $10 \mathrm{mM}$ citric sodium (pH 6.0) for 15 min to unmask antigens. The sections were rinsed in PBS and then incubated with the primary anti-CD31 goat polyclonal antibody (1:100; cat. no. sc-1506; Santa Cruz Biotechnology, Inc., Santa Cruz, CA, USA) for $1 \mathrm{~h}$ at room temperature and washed with TBS. Biotinylated anti-goat IgG (1:100; cat. no. B-205; Zhongshan Golden Bridge Biotechnology Co., Ltd.) was applied for $30 \mathrm{~min}$ at room temperature. Color was developed following $10 \mathrm{~min}$ of incubation with the 3,3'-diaminobenzidine. For MVD quantification, the number of CD31-positive vessels/x100 field were

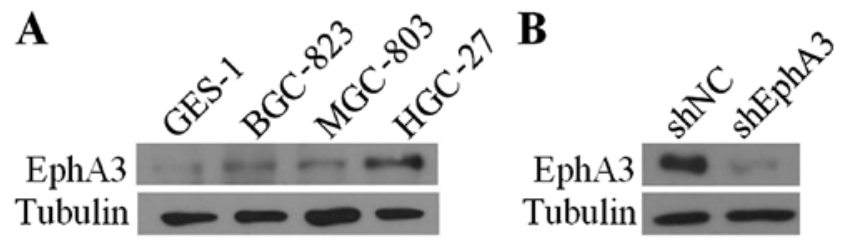

Figure 1. Knockdown of the expression of EphA3 by lentivirus-mediated shRNA. (A) EphA3 protein expression in gastric epithelial mucosa cells and 3 distinct differentiated GC cell lines was analyzed by western blotting. (B) EphA3 protein expression in shNC and shEphA3-HGC-27 cells was analyzed by western blotting. shNC, shNC-HGC-27.

counted in 5 randomly selected fields. The data are represented as the number of CD31-positive microvessels/x 200 microscopic field.

Statistical analysis. Statistical analysis was performed using SPSS 13.0 (SPSS, Inc., Chicago, IL, USA) and the results were analyzed statistically using Student's t-test for comparisons between two groups. $\mathrm{P}<0.05$ was considered to indicate a statistically significant difference.

\section{Results}

Comparison of EphA3 expression levels. To investigate the molecular effects of EphA3 in GC, we initially examined the expression level of EphA3 in GC cells compared with the human gastric epithelial mucosa cells GES-1 using western blot analysis. The results revealed that EphA3 expression was upregulated in three distinct differentiated GC cell lines MGC-803, BGC-823 and HGC-27 (Fig. 1A). With the highest levels of EphA3 expression, the HGC-27 cell line was selected to knock down endogenous EphA3. To establish stable cell lines, EphA3 expression was effectively inhibited in HGC-27 cells infected with lentiviral particles containing EphA3 shRNA compared to those infected with control shRNA vector (Fig. 1B).

EphA3 knockdown inhibits the growth of HGC-27 cells in vitro and in vivo. To determine whether EphA3 knockdown affected the proliferation of HGC-27 cells, cell growth was determined using CCK-8 and colony formation assays. EphA3 knockdown in HGC-27 cells significantly impaired cellular proliferation compared with the control cells (Fig. 2A). Furthermore, colony formation capacity was also reduced by EphA3 knockdown (Fig. 2B). We further examined the effects of EphA3 on tumor growth using a subcutaneous xenograft model of HGC-27 cells in mice. EphA3 knockdown did not induce any toxicity in mice, however, we observed a significant decrease $(39.1 \%)$ in mean tumor volume in nude mice implanted with shEphA3-HGC-27 cells compared with that implanted with shNC-HGC-27 xenografts (Fig. 2C and D). These results indicated that loss of EphA3 expression inhibited the proliferation of HGC-27 in vitro and in vivo.

EphA3 knockdown inhibits tube formation and angiogenesis in vitro and in vivo through the regulation of the expression of VEGF. In our previous study, we provided clinical evidence that EphA3 expression was significantly and positively 


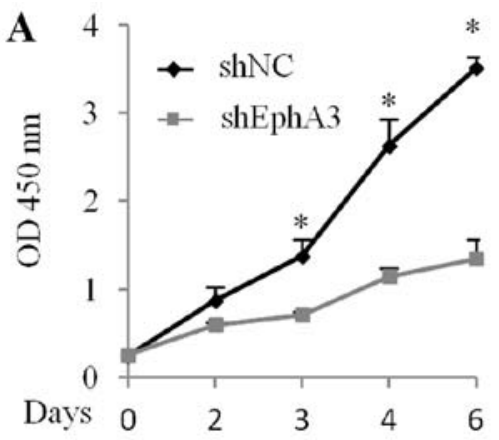

C

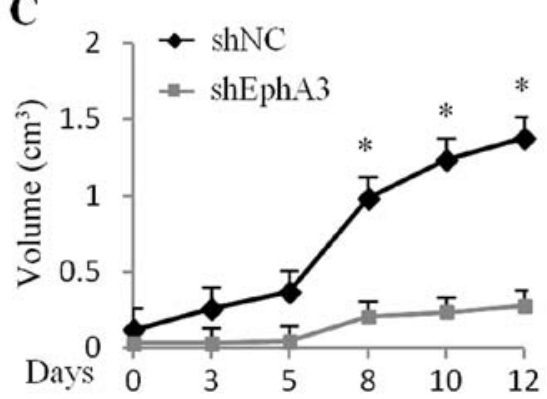

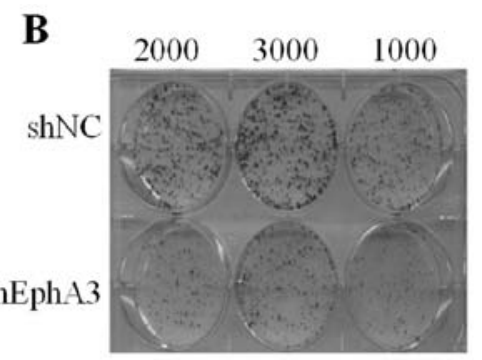

D

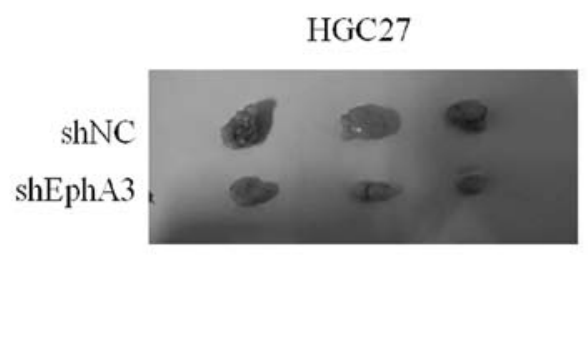

Figure 2. EphA3 knockdown and tumor growth of HGC-27 cells. (A) A CCK-8 assay was used to estimate cell proliferation at different time-points. Cell proliferation was significantly suppressed by EphA3 knockdown in HGC-27 cells. (B) Effect of EphA3 knockdown on colony formation. EphA3 knockdown reduced colony formation capacity of HGC-27 cells compared with shNC-HGC-27 cells. (C) The effect of EphA3 knockdown on tumor growth in vivo; $5 \times 10^{6}$ of the shNC or shEphA3-HGC-27 cells were injected s.c. into the right flanks of the nude mice ( $\mathrm{n}=3$ per group). The tumor volume was monitored at indicated time-points and tumor growth curves are presented as the mean \pm SD. (D) Representative images of tumors were captured after the shNC and shEphA3-HGC-27 nude mice were euthanized at day 21. ${ }^{*} \mathrm{P}<0.05$ compared with control shNC. shNC, shNC-HGC-27.

associated with the expression of VEGF and MVD status. These findings indicated that EphA3 may be involved in the angiogenic process of GC. To obtain direct evidence on whether EphA3 regulated the expression of VEGF and was involved in angiogenesis, an ELISA assay was performed to determine secreted VEGF-A in the CM of HGC-27 cells. The results revealed that EphA3 knockdown reduced VEGF secretion in HGC-27 cells (Fig. 3A), indicating that EphA3 may be involved in regulating the formation of new blood vessels in endothelial cells. To determine whether the CM from shNC-HGC-27 and shEphA3-HGC-27 cells could induce the tube formation of HUVECs, we performed a tube formation assay in growth factor-reduced Matrigel in vitro. We observed that the CM from shNC-HGC-27 and shEphA3-HGC-27 cells was able to significantly induce HUVEC tube formation in 6-h incubation time-periods. However, tube formation of HUVECs was significantly reduced in CM from the shEphA3-HGC-27 cells (Fig. 3B). Since the migration and invasion of endothelial cells through basement membranes is a crucial step in the development of new blood vessels, we assessed the effect of CM from HGC-27 on endothelial cell HUVEC migration. As displayed in Fig. 3C, HUVECs cultured with the CM from the NC-HGC-27 cells migrated faster compared to the shEphA3-HGC-27 cells.

To further examine the effect of EphA3 on angiogenesis in vivo, we identified the MVD status in HGC-27 cell xenograft nude mice by immunohistochemical staining with anti-CD31 antibody. EphA3 knockdown significantly reduced microvessel formation in the shEphA3-HGC-27 tumors relative to that in the shNC-HGC-27 control (Fig. 3D). These results indicated that blockade of EphA3 activity suppressed
VEGF expression and impaired the angiogenic phenotype of GC HGC-27 cells in vitro and in vivo.

The VEGF signaling pathway plays a crucial role in tumor angiogenesis, thereby promoting endothelial cell proliferation, migration and capillary tube formation. To further elucidate the role of VEGF in EphA3-mediated angiogenesis modulation, neutralization of the VEGF antibody was used to antagonize the functions of VEGF. We found that when VEGF neutralizing antibody was used to neutralize VEGF in the culture supernatants of HUVEC cells from the CM of shNC-HGC-27 and shEphA3-HGC-27 cells, the tube formation and migration of the HUVEC cells induced by EphA3 were markedly attenuated in vitro (Fig. 3E and F). These results indicated that EphA3-dependent VEGF expression played an important role in the process of EphA3-regulated angiogenesis.

The JAK2/STAT3 signaling pathway is involved in EphA3-mediated VEGF expression and angiogenesis. Our present study indicated that knockdown of EphA3 expression reduced the tube formation and migration of HUVECs and tumor angiogenesis by inhibiting the secretion of VEGF. To further understand the regulation of VEGF expression by EphA3, we investigated the main signaling pathways related to the expression of VEGF. Accumulating evidence is defining STAT3 signaling as an important pathway for upregulation of the expression of VEGF and tumor angiogenesis. In order to investigate whether changes in EphA3 levels would affect the phosphorylation of STAT3, we assessed the changes in STAT3 phosphorylation by western blotting following knockdown of EphA3 in HGC-27 cells. Our data indicated that after EphA3 knockdown, p-STAT3 (Tyr705) levels were 

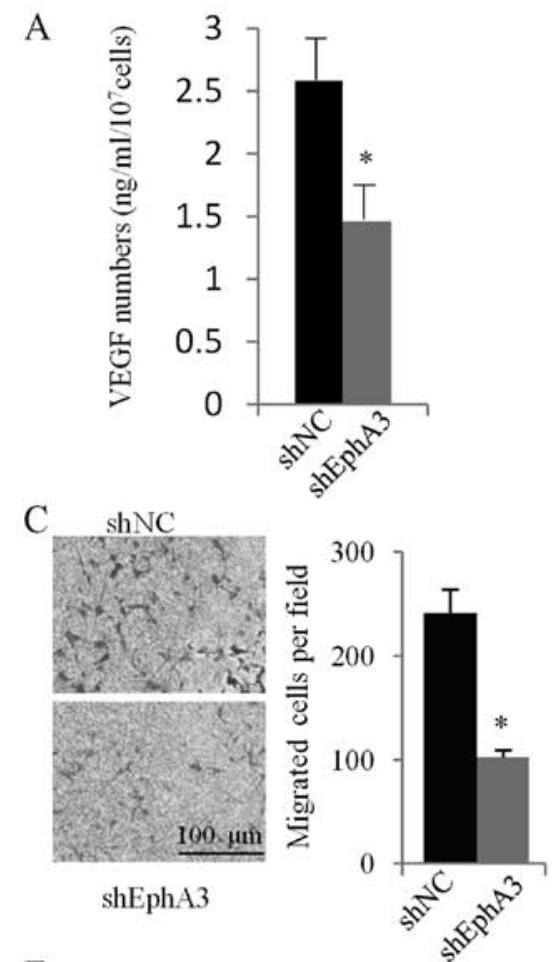

$\mathrm{D}$

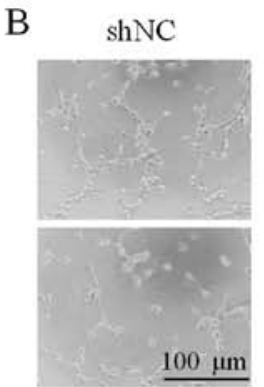

ShEphA3

$\operatorname{shNC}$

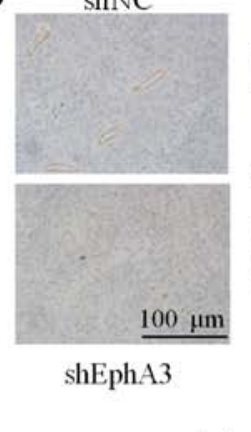

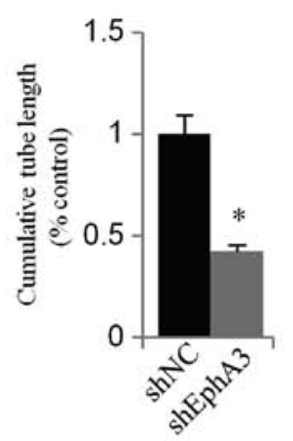

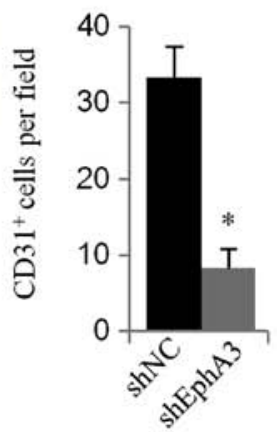

$\mathrm{E}$

$\operatorname{shNC}$

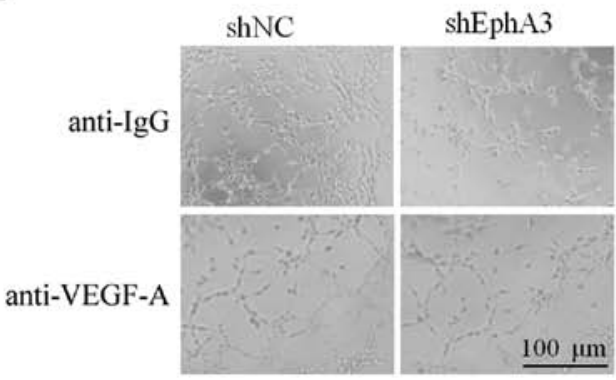

F

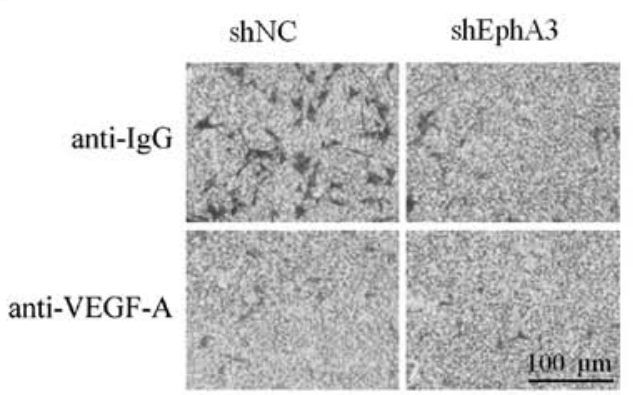

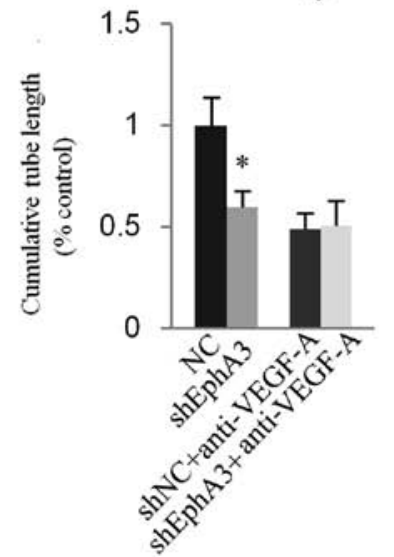

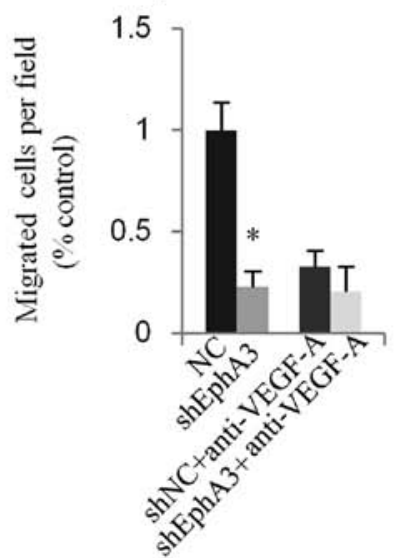

Figure 3. EphA3 knockdown inhibits the tube formation and angiogenesis in vitro and in vivo through the regulation of the expression of VEGF. (A) Secreted VEGF that was released into the CM of shNC and shEphA3-HGC-27 was determined by ELISA. Results were normalized to the cell number and expressed as $\mathrm{ng} / \mathrm{ml} / 10^{7}$ cells. $\mathrm{n}=3$. ${ }^{*} \mathrm{P}<0.05$ compared with control shNC. (B) Tube formation ability of HUVECs cultured in the CM from shNC and shEphA3-HGC-27 cells. Representative images of tube-like structures (left panel) and mean numbers of tube length (right panel) at an x100 magnification are shown. Data are from 3 independent experiments. " $\mathrm{P}<0.05$ compared with control shNC. (C) Migration of HUVECs cultured in CM from shNC and shEphA3-HGC-27 cells was performed in a Transwell chamber with $8.0-\mu \mathrm{m}$ pore size. Representative images of HUVECs that migrated through the membrane (left panel) and the number of cells that migrated through the membrane/field (right panel) are presented. Values are expressed as the mean $\pm \mathrm{SD}$. $\mathrm{n}=3$. "P $<0.05$ compared with control shNC. (D) Tumor sections from shNC and a shEphA3-HGC-27 cell xenograft model were used. Representative cases of immunohistochemical staining with CD31 (left panel) and quantitative analysis of CD31-positive blood vessels (right panel) are displayed. Values are expressed as the mean \pm SD. $n=3$. "P $<0.05$ compared with control shNC. (E and F) Following the addition of neutralizing antibody $(2 \mu \mathrm{g} / \mathrm{ml})$ to VEGF-A or IgG into CM, tube formation and a migration assay of HUVEC cells were performed as described in B and C. Scale bar, $100 \mu \mathrm{M}$. VEGF, vascular endothelial growth factor; CM, conditioned media; HUVECs, human umbilical vein endothelial cells; shNC, shNC-HGC-27. 
A

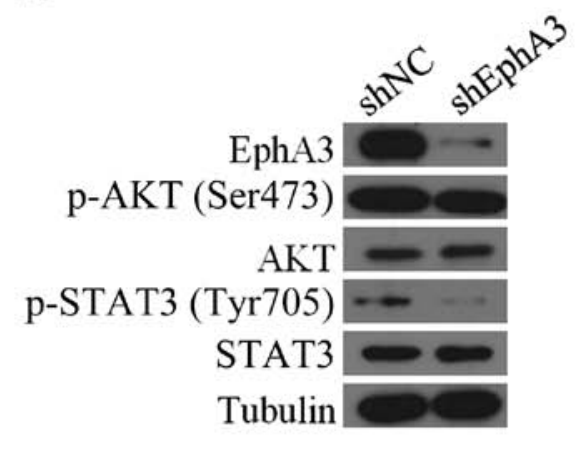

C

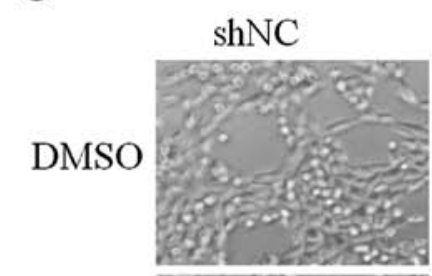

shEphA3

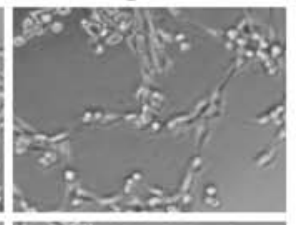

WP1066
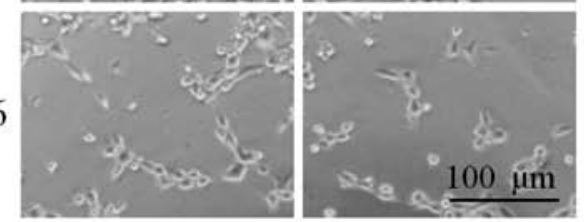

D

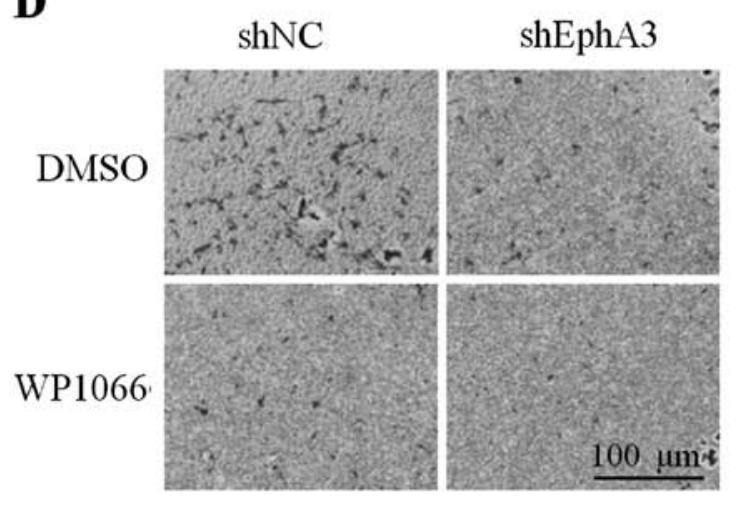

B
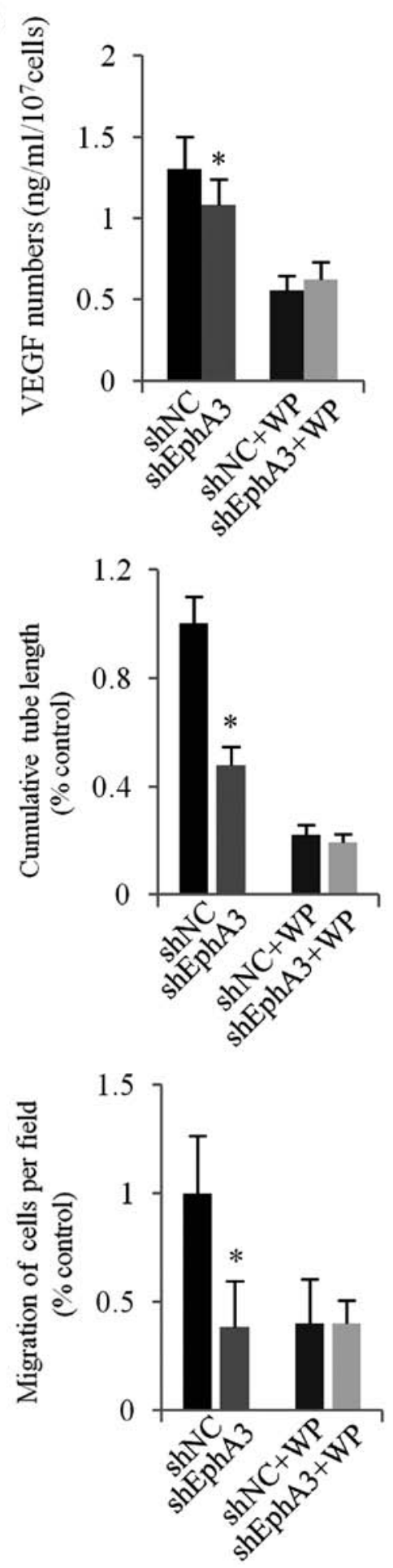

Figure 4. STAT3 signaling pathway is involved in EphA3-mediated VEGF expression and angiogenesis. (A) p-Stat3(Tyr705), Stat3, p-AKT(Ser473), AKT and EphA3 protein expression in shNC and shEphA3-HGC-27 cells was analyzed by western blotting. (B) Secreted VEGF that was released into the CM from shNC and shEphA3-HGC-27 cells treated with DMSO or WP1066 $(5 \mu \mathrm{M})$ was determined by ELISA. Results were normalized to the cell number and expressed as $n g / m l / 10^{7}$ cells. $n=3$. ${ }^{*} \mathrm{P}<0.05$ compared with control shNC. (C) Tube formation ability of HUVECs cultured in CM from shNC and shEphA3-HGC-27 cells treated with DMSO or WP1066 $(5 \mu \mathrm{M})$. Representative images of tube-like structures (left panel) and mean numbers of tube length (right panel) at an x100 magnification are presented. Data are from 3 independent experiments. "P<0.05 compared with the control shNC. (D) Migration of HUVECs cultured in CM from shNC and shEphA3-HGC-27 cells treated with DMSO or WP1066 $(5 \mu \mathrm{M})$ was performed in a Transwell chamber with 8.0- $\mu \mathrm{m}$ pore size. Representative images of HUVECs that migrated through the membrane (left panel) and the number of cells that migrated through the membrane/field (right panel) are presented. Values are expressed as the mean $\pm \mathrm{SD}$. $\mathrm{n}=3$. ${ }^{*} \mathrm{P}<0.05$ compared with the control shNC. Scale bar, $100 \mu \mathrm{M}$. VEGF, vascular endothelial growth factor; CM, conditioned media; HUVECs, human umbilical vein endothelial cells; shNC, shNC-HGC-27.

significantly decreased (Fig. 4A). To examine whether STAT3 activity was involved in EphA3-mediated VEGF expression and angiogenesis, JAK2/STAT3 inhibitor WP1066 was used to inhibit the JAK2/STAT3 signaling pathway of HGC-27 cells 
before collection of CM. The results revealed that pretreatment of cells with WP1066 reduced the expression of VEGF and the difference of the VEGF level between shNC-HGC-27 and shEphA3-HGC-27 was also significantly decreased (Fig. 4B). In addition, EphA3-mediated HUVEC migration and tube formation were also attenuated by pretreatment with WP1066 (Fig. 4C and D). Collectively, EphA3 appeared to act through the JAK2/STAT3 signaling pathway to enhance the expression of VEGF and angiogenesis in human GC HGC-27 cells.

\section{Discussion}

In GC, growing evidence has revealed that some Eph receptors were aberrantly expressed and were associated with cancer progression, metastasis and poor prognosis. For example, the EphA1 protein has been significantly associated with the depth of invasion of GC (13). The expression of EphA2 was revealed to be upregulated in GC compared to that in normal mucosa and was consistent with tumor TNM stage. EphA2 knockdown could inhibit GC cell proliferation and invasion in vitro and in vivo (14). Eph $\mathrm{A} 7$ overexpression has been observed in gastric carcinoma specimens and is related to the pathogenesis and development of GC (15).

EphA3 was first identified in a pre-B acute lymphoblastic leukemia cell line and has also been reported to be expressed in sarcomas, lung cancer, melanoma and glioblastoma. EphA3 is currently one of the most promising therapeutic targets (9). Numerous studies support multiple tumor-promoting roles for EphA3 in a range of solid and hematological cancers, including glioblastoma-initiating cells and leukemic stem cells. Recently, we observed that in GC the upregulated expression of EphA3 was positively associated with the expression of VEGF, MVD as well as poor prognosis (12). Thus, EphA3 may play important roles in angiogenesis and prognosis of GC. However, the precise role of EphA3 in GC progression remains unknown.

To elucidate the biological functions of EphA3 in GC tumorigenesis, we knocked down EphA3 expression in HGC-27 cells. Knockdown of EphA3 markedly reduced cell viability and proliferation in vitro and inhibited tumor growth in in vivo assays. Furthermore, the present study confirmed that Eph A3 contributed to tumor angiogenesis in GC and was mediated by STAT3-dependent expression of VEGF.

Angiogenesis, a process of neovascular formation, is essential for the progression and metastasis of tumors (16). In recent years, ephrin ligands with Eph receptors have been identified as contributors to tumor angiogenesis. Studies revealed that EphA2-null ECs and EphA2-deficient mice failed to undergo vascular assembly in vitro and angiogenesis in vivo (17). Additionally, studies have shown that injections of soluble EphA2 and EphA3-Fc receptors or EphB4 extracellular domains into tumor-bearing mice were able to inhibit tumor angiogenesis and growth in vivo $(11,18,19,20)$. Two recent studies revealed ephrin-B2 as a key regulator in VEGFR-2 and VEGFR-3 endocytosis, with consequences for the development and tumor angiogenesis $(21,22)$. Recently, a study in multiple myeloma (MM) demonstrated that EphA3 was overexpressed in bone marrow ECs, and that EphA3 knockdown inhibited the adhesion, migration and angiogenesis in vitro of ECs of
MM patients $(23,24)$. Our present study provided evidence that EphA3 knockdown in HGC-27 cells inhibited tube formation and migration of EC in vitro. We also revealed that vessel density was lower in HGC-27 tumors following knockdown of EphA3 compared with the control group. Collectively, our results demonstrated a role for EphA3 in promoting angiogenesis in GC.

The balance of various proangiogenic stimulators and angiogenesis inhibitors regulates the angiogenic process. Tumor angiogenesis begins with the activation of ECs by a few specific angiogenic factors, among which VEGF is a potent angiogenic molecule responsible for tumor progression and metastasis through its enhancement of angiogenesis $(25,26)$. In hepatocellular carcinoma cells, EphA3 knockdown was found to decrease the invasiveness of cells by regulating VEGF protein expression and proteolytic activity (27). In the present study, the effect of EphA3 on VEGF secretion and expression in GC cells was assessed by ELISA. Our data revealed that EphA3 knockdown inhibited VEGF production in HGC-27 cells. Furthermore, VEGF was involved in the effects of EphA3 on tube formation and migration in HUVECs cultured in vitro since a neutralizing anti-VEGF antibody reduced angiogenesis. These results demonstrated that EphA3 played a role in tumor angiogenesis by regulating VEGF expression in HGC-27 cells.

It is well recognized that VEGF is regulated by many signaling pathways, such as the ERK1/2 and JAK/STAT3 pathways $(28,29)$. STAT3 is a critical transcription activator and has been demonstrated to be very important for cancer progression. Accumulating evidence supports a pivotal role of constitutive Stat 3 activity in upregulating VEGF expression and tumor angiogenesis $(30,31)$. Many studies have concentrated on STAT3 as a potential target for cancer therapy and found that STAT3 inhibition effectively blocked production of VEGF and tumor angiogenesis $(32,33)$. Previously, Eph family members, including EphA1, EphA5, EphB2, EphB3 and EphB4, have been reported to constitutively activate STAT3 (34). A study has shown that ephrinB1 interacted with STAT3 in a tyrosine phosphorylation-dependent manner, resulting in the phosphorylation and enhanced transcriptional activation of STAT3 (35). This study revealed that EphA3 knockdown negatively regulated STAT3 activity. Blocking the JAK/STAT3 signaling pathway with WP1066 effectively impaired the promotion of VEGF expression, HUVEC tube formation and migration caused by EphA3 expression. These results indicated that the effects of EphA3 on angiogenesis of HGC-27 cells were dependent on STAT3-mediated VEGF production.

Despite the challenges presented by the complex biology of the Eph receptors and ephrins, this system still represents promising new therapeutic targets for the inhibition of angiogenesis and tumorigenesis. In the present study, the results demonstrated that EphA3 promoted HGC-27 tumor cell growth and angiogenesis through the STAT3/VEGF pathway, indicating that EphA3 may be an effective indicator for prognosis and a potential target for GC therapy.

\section{Acknowledgements}

Not applicable. 


\section{Funding}

The present study was supported by the National Natural Science Foundation of China (nos. 81372740, 81372770, 81672604 and 81772750) and the National Key Research and Development Program of China (no. 2016YFC1202402).

\section{Availability of data and materials}

All data generated or analyzed during this study are included in this published article.

\section{Authors' contributions}

SHL and BW designed the study; JW wrote the manuscript; JGZ revised the manuscript; XYL and JW performed the experiments including in vitro experiments, xenograft models, immunohistochemical staining and statistical analysis; FH and PW participated in cell culture, cell transfection and western blot assay. All authors read and approved the manuscript and agree to be accountable for all aspects of the research in ensuring that the accuracy or integrity of any part of the work are appropriately investigated and resolved.

\section{Ethics approval and consent to participate}

All animal studies conformed to the relevant regulatory standards and were approved by the Institutional Animal Care Committee of Beijing Institute of Biotechnology.

\section{Patient consent for publication}

Not applicable.

\section{Competing interests}

The authors declare that they have no competing interests.

\section{References}

1. Ferlay J, Soerjomataram I, Dikshit R, Eser S, Mathers C, Rebelo M, Parkin DM, Forman D and Bray F: Cancer incidence and mortality worldwide: Sources, methods and major patterns in GLOBOCAN 2012. Int J Cancer 136: E359-E386, 2015.

2. Chen W, Zheng R, Baade PD, Zhang S, Zeng H, Bray F, Jemal A, $\mathrm{Yu}$ XQ and He J: Cancer statistics in china.2015. CA Cancer J Clin 66: 115-132, 2016

3. Nagini S: Carcinoma of the stomach: A review of epidemiology, pathogenesis, molecular genetics and chemoprevention. World J Gastrointest Oncol 4: 156-169, 2012.

4. Pasquale EB: Eph-ephrin promiscuity is now crystal clear. Nat Neurosci 7: 417-418, 2004.

5. Pasquale EB: Eph receptor signalling casts a wide net on cell behavior. Nat Rev Mol Cell Biol 6: 462-475, 2005.

6. Pasquale EB: Eph receptors and ephrins in cancer: Bidirectional signalling and beyond. Nat Rev Cancer 10: 165-180, 2010.

7. Valsesia A, Rimoldi D, Martinet D, Ibberson M, Benaglio P, Quadroni M, Waridel P, Gaillard M, Pidoux M, Rapin B, et al: Network-guided analysis of genes with altered somatic copy number and gene expression reveals pathways commonly perturbed in metastatic melanoma. PLoS One 6: e18369, 2011.

8. Wood LD, Calhoun ES, Silliman N, Ptak J, Szabo S, Powell SM Riggins GJ, Wang TL, Yan H, Gazdar A, et al: Somatic mutations of GUCY2F, EPHA3, and NTRK3 in human cancers. Hum Mutat 27: 1060-1061, 2006.
9. Janes PW, Slape CI, Farnsworth RH, Atapattu L, Scott AM and Vail ME: EphA3 biology and cancer. Growth Factors 32: 176-189, 2014.

10. Day BW, Stringer BW, Al-Ejeh F, Ting MJ, Wilson J, Ensbey KS, Jamieson PR, Bruce ZC, Lim YC, Offenhäuser C, et al: EphA3 maintains tumorigenicity and is a therapeutic target in glioblastoma multiforme. Cancer Cell 23: 238-248, 2013.

11. Brantley DM, Cheng N, Thompson EJ, Lin Q, Brekken RA, Thorpe PE, Muraoka RS, Cerretti DP, Pozzi A, Jackson D, et al: Soluble Eph A receptors inhibit tumor angiogenesis and progression in vivo. Oncogene 21: 7011-7026, 2002.

12. Xi HQ, Wu XS, Wei B and Chen L: Aberrant expression of EphA3 in gastric carcinoma: Correlation with tumor angiogenesis and survival. J Gastroenterol 47: 785-794, 2012.

13. Wang J, Dong Y, Wang X, Ma H, Sheng Z, Li G, Lu G, Sugimura $\mathrm{H}$ and Zhou X: Expression of EphA1 in gastric carcinomas is associated with metastasis and survival. Oncol Rep 24: 1577-1584, 2010.

14. Nakamura R, Kataoka H, Sato N, Kanamori M, Ihara M, Igarashi H, Ravshanov S, Wang YJ, Li ZY, Shimamura T, et al: EPHA2/EFNA1 expression in human gastric cancer. Cancer Sci 96: 42-47, 2005

15. Wang J, Li G, Ma H, Bao Y, Wang X, Zhou H, Sheng Z, Sugimura H, Jin J and Zhou X: Differential expression of EphA7 receptor tyrosine kinase in gastric carcinoma. Hum Pathol 38: 1649-1656, 2007.

16. Carmeliet P and Jain RK: Angiogenesis in cancer and other diseases. Nature 407: 249-257, 2000.

17. Brantley-Sieders DM, Fang WB, Hicks DJ, Zhuang G, Shyr Y and Chen J: Impaired tumor microenvironment in EphA2-deficient mice inhibits tumor angiogenesis and metastatic progression. FASEB J 19: 1884-1886, 2005.

18. Dobrzanski P, Hunter K, Jones-Bolin S, Chang H, Robinson C, Pritchard S, Zhao H and Ruggeri B: Antiangiogenic and antitumor efficacy of EphA2 receptor antagonist. Cancer Res 64: 910-919, 2004.

19. Kertesz N, Krasnoperov V, Reddy R, Leshanski L, Kumar SR Zozulya S and Gill PS: The soluble extracellular domain of EphB4 (sEphB4) antagonizes EphB4-EphrinB2 interaction, modulates angiogenesis, and inhibits tumor growth. Blood 107: 2330-2338, 2006.

20. Martiny-Baron G, Korff T, Schaffner F, Esser N, Eggstein S, Marmé D and Augustin HG: Inhibition of tumor growth and angiogenesis by soluble EphB4. Neoplasia 6: 248-257, 2004.

21. Sawamiphak S, Seidel S, Essmann CL, Wilkinson GA, Pitulescu ME, Acker T and Acker-Palmer A: Ephrin-B2 regulates VEGFR2 function in developmental and tumour angiogenesis. Nature 465: 487-491, 2010.

22. Wang Y,Nakayama M, Pitulescu ME, Schmidt TS, Bochenek ML, Sakakibara A, Adams S, Davy A, Deutsch U, Lüthi U, et al: Ephrin-B2 controls VEGF-induced angiogenesis and lymphangiogenesis. Nature 465: 483-486, 2010.

23. Caivano A, La Rocca F, Laurenzana I, Annese T, Tamma R, Famigliari U, Simeon V, Trino S, De Luca L, Villani O, et al: Epha3 acts as proangiogenic factor in multiple myeloma. Oncotarget 8: 34298-34309, 2017.

24. La Rocca F, Airoldi I, Di Carlo E, Marotta P, Falco G, Simeon V, Laurenzana I, Trino S, De Luca L, Todoerti K, et al: EphA3 targeting reduces in vitro adhesion and invasion and in vivo growth and angiogenesis of multiple myeloma cells. Cell Oncol (Dordr) 40: 483-496, 2017.

25. Iruela-Arispe ML and Dvorak HF: Angiogenesis: A dynamic balance of stimulators and inhibitors. Thromb Haemost 78: 672-677, 1997.

26. Nicosia RF: What is the role of vascular endothelial growth factor-related molecules in tumor angiogenesis? Am J Pathol 153: 11-16, 1998.

27. Lu CY, Yang ZX, Zhou L, Huang ZZ, Zhang HT, Li J, Tao KS and Xie BZ: High levels of EphA3 expression are associated with high invasive capacity and poor overall survival in hepatocellular carcinoma. Oncol Rep 30: 2179-2186, 2013.

28. Milanini J, Viñals F, Pouysségur J and Pagès G: p42/p44 MAP kinase module plays a key role in the transcriptional regulation of the vascular endothelial growth factor gene in fibroblasts. J Biol Chem 273: 18165-19172, 1998.

29. Niu G, Wright KL, Huang M, Song L, Haura E, Turkson J, Zhang S, Wang T, Sinibaldi D, Coppola D, et al: Constitutive Stat3 activity up-regulates VEGF expression and tumor angiogenesis. Oncogene 21: 2000-2008, 2002. 
30. Huang S: Regulation of metastases by signal transducer and activator of transcription 3 signaling pathway: Clinical implications. Clin Cancer Res 13: 1362-1366, 2007.

31. Wei D, Le X, Zheng L, Wang L, Frey JA, Gao AC, Peng Z, Huang S, Xiong HQ, Abbruzzese JL and Xie K: Stat3 activation regulates the expression of vascular endothelial growth factor and human pancreatic cancer angiogenesis and metastasis. Oncogene 22: 319-329, 2003

32. Cheong JH, Hong SY, Zheng Y and Noh SH: Eupatilin inhibits gastric cancer cell growth by blocking STAT3-mediated VEGF expression. J Gastric Cancer 11: 16-22, 2011.

33. Xu Q, Briggs J, Park S, Niu G, Kortylewski M,Zhang S, Gritsko T, Turkson J, Kay H, Semenza GL, et al: Targeting Stat3 blocks both HIF-1 and VEGF expression induced by multiple oncogenic growth signaling pathways. Oncogene 24: 5552-5560, 2005.
34. Uan ZL, Guan YJ, Wang L, Wei W, Kane AB and Chin YE: Central role of the threonine residue within the $\mathrm{p}+1$ loop of receptor tyrosine kinase in STAT3 constitutive phosphorylation in metastatic cancer cells. Mol Cell Biol 24: 9390-9400, 2004.

35. Bong YS, Lee HS, Carim-Todd L, Mood K, Nishanian TG, Tessarollo L and Daar IO: ephrinB1 signals from the cell surface to the nucleus by recruitment of STAT3. Proc Natl Acad Sci USA 104: 17305-17310. 